\title{
Acceptance of Provider Initiated HIV Testing and Counseling among Tuberculosis Patients in East Wollega Administrative Zone, Oromia Regional State, Western Ethiopia
}

\author{
Wakjira Kebede, ${ }^{1}$ Fikru Keno, ${ }^{2}$ Temesgen Ewunetu, ${ }^{1}$ and Gutu Mamo ${ }^{3}$ \\ ${ }^{1}$ Department of Medical Laboratory Sciences and Pathology, College of Public Health and Medical Sciences, \\ Jimma University, Jimma, Ethiopia \\ ${ }^{2}$ Oromia Regional Social and Labor Affairs Agency, Addis Ababa, Ethiopia \\ ${ }^{3}$ Department of Pharmacy, College of Public Health and Medical Sciences, Nekemte Hospital, Jimma, Ethiopia
}

Correspondence should be addressed to Wakjira Kebede; dandiwk2004@gmail.com

Received 12 November 2013; Revised 23 December 2013; Accepted 13 February 2014; Published 20 March 2014

Academic Editor: Brian Eley

Copyright (C) 2014 Wakjira Kebede et al. This is an open access article distributed under the Creative Commons Attribution License, which permits unrestricted use, distribution, and reproduction in any medium, provided the original work is properly cited.

\begin{abstract}
Human immunodeficiency virus (HIV) is a powerful risk factor for the development of tuberculosis. This study assessed the acceptance and associated factors that can affect provider initiated HIV testing and counseling (PITC) among tuberculosis patients at the East Wollega administrative zone, Oromia regional state, western Ethiopia, from January to August, 2010. A single population proportion formula is used to calculate the total sample size of 406 and the cluster sampling technique was used to select 13 health centers that provide PITC services. The sample size was proportionally allocated to each health center. The study participants were selected using a simple random sampling technique using the lottery method. Structured questionnaire was used for collection of sociodemographic data. From the total of study subjects, 399 (98.2\%) TB patients were initiated for HIV test and 369 (92.5\%) patients accepted the initiation. Of those, 353 (95.5\%) patients had taken HIV test and received their results. According to the reviewed documents, the prevalence of HIV among tuberculosis (TB) patients in the study area was 137 (33.7\%). The logistic regression result showed the PITC was significantly associated with their knowledge about HIV (AOR = 3.22, 95\% CI: 1.3-7.97), selfperceived risk ( $\mathrm{AOR}=2.93,95 \% \mathrm{CI}: 1.12-7.66)$, educational status ( $\mathrm{AOR}=3.51,95 \% \mathrm{CI}: 1.13-10.91)$, and knowledge on transmission of HIV/AIDS (AOR $=7.56,95 \%$ CI: 1.14-40.35) which were significantly associated with the acceptance of PITC among TB patients. Therefore, this study's results showed, the prevalence of HIV among TB patient was high; to enhance the acceptance of PITC among TB patients, health extension workers must provide health education during home-to-home visiting. TB treatment supervisors also provide counseling intensively for all forms of TB patients during their first clinical encounter.
\end{abstract}

\section{Introduction}

World Health Organization (WHO) estimates that one-third of the world's population is infected with Mycobacterium tuberculosis, resulting in an estimated nearly 8.7 million incident cases of tuberculosis (TB) and 1.4 million deaths in 2012. TB has been closely linked to human immunodeficiency virus (HIV) and is believed to account for $50-80 \%$ in parts of sub-Saharan Africa for 500,000 deaths; $25 \%$ of the total deaths occur in people living with HIV [1-3]. Worldwide, TB is the most common opportunistic infection affecting HIV-seropositive and it remains the most common cause of death and major threat in terms of its personal, family, and socioeconomic consequences [4].

WHO Global report of 2012 ranked Ethiopia as the seventh among the highest TB burden countries in the world, with an estimated incidence and prevalence of all forms of TB cases being 258, 237/100,000 population, respectively [5]. Ethiopia Ministry of Health Hospital statistics data show that TB is the third leading cause of outpatient morbidity and mortality and the fourth leading cause of hospital admission [6].

HIV is the main reason for failure to meet TB control targets in high HIV settings, because HIV infection has 
contributed to progressive decline in immune response and pathogenesis of TB, increasing the risk of coinfection leading to more frequent extrapulmonary disease $[4,7]$. The risk of reactivating $\mathrm{TB}$ infection in $\mathrm{HIV}$ infected persons is $7-10 \%$ per year compared with $10 \%$ lifetime risk in those serum negative persons. In order to control TB in high HIV settings, the Stop TB strategy needs collaborative TB/HIV activities in the country $[4,5,8]$.

Worldwide only $20 \%$ of people living with HIV know their status, and only one in four people estimated to be living with HIV and TB is detected and treated for both diseases [3]. In sub-Saharan Africa only $10-12 \%$ of people living with HIV know their serostatus [9]. In Ethiopia most of the adult segment of the population (95\%) does not know their HIV status [10]. And ART has been accessed by only $13 \%$ of those who need ART [11].

Currently, acceptability of provider initiated HIV testing and counseling (PITC) among TB patients varies from place to place. In Ethiopia the acceptability of PITC may be influenced by different associated factors like fear of stigma and discrimination associated with having TB and HIV, sociodemographic factors, knowledge related to TB/HIV, the general perception of HIV infection, and attitude towards PITC may affect the acceptance and knowledge of TB patients. PITC is one of the main intervention areas to fight against failure to meet $\mathrm{TB}$ control targets in areas where HIV prevalence was high, particularly, for those coinfected patients with TB and HIV.

Therefore, the aim of this study was to assess the acceptance of PITC and factors affecting PITC acceptance among TB patients. Furthermore, the results of this study help to design and practice appropriate interventions that decrease the burden of HIV on TB patient by enhancing the utilization of PITC service and are also important to improve treatment outcome by preventing other infectious diseases and decrease life loss of AIDS cases secondary to tuberculosis.

\section{Materials and Methods}

An institution based, cross-sectional study was conducted from July to August 2010 among adult tuberculosis patients in the East Wollega administrative zone, Oromia regional state, western Ethiopia. It is located at $262 \mathrm{KMs}$ from Jimma University and $331 \mathrm{KMs}$ from Addis Ababa (the capital city). The expected population of the zone for 2005 was estimated to be $1,647,576$. The zone was divided into 21 administrative districts. There were 2 hospitals, one zonal hospital and one district hospital, 15 health centers, 82 health stations, and 107 health posts; all were public [12]. All tuberculosis patients in selected health centers registered for directly observed treatment short course (DOTS) from October 8, 2009, to August 18, 2010, and TB cases encountered during the data collection period were included in the study and those TB patients below the age of 18 years and those critically ill patients are excluded from the study.

A single population proportion formula is used to calculate the total sample size of 406 and cluster sampling technique was used to select woredas based on their geographical location. The health center was selected from the total health centers using a simple random sampling technique. The number of participants was proportionally allocated to each health center. The identification number (ID number) of TB patients at each health center was written on a piece of paper and coiled and then the actual number of 406 study units was selected by using the lottery method. A structured questionnaire was used to obtain sociodemographic information (residence, ethnicity, sex, age, religion, marital status, educational status, occupation, and family size).

The study protocol was reviewed and approved by the Institutional Ethical Review Board of the Jimma University. The objective of the study was explained to the participants and verbal consent was obtained from each participant. Confidentiality of the result was kept by coding patient information. The data were checked for their completeness before entering for analysis; then all the data from study participants were imported into excel and analyzed by SPSS version 20 program software. Odds ratio, 95\% confidence interval, and both bivariate and multivariate logistic regression were used to test the association between the outcome and independent variables.

\section{Results}

3.1. Sociodemographic. A total of $406 \mathrm{~TB}$ patients were included in the study and are with a $100 \%$ response rate. Of the total, $234(57.6 \%)$ of the study participants were male with nearly 1.36 to 1 sex ratio. The mean age of the study populations was 35 years with 1.2 standard deviation (SD); more than half $(68.2 \%)$ of the interviewed religions were Orthodox, followed by Protestant 120 (29.6\%); 391 (96.3\%) and $278(68.5 \%)$ were identifying themselves as an Oromo ethnic group and live in urban areas, respectively. Marriage characteristics, educational status, family size, and monthly income per month were identified (Table 1).

3.2. Knowledge about TB/HIV/AIDS. Almost all study participants $404(99.5 \%)$ believed that TB is a curable disease. $306(75.4 \%)$ of the respondents believed that a person who lives with HIV/AIDS is at risk of acquiring tuberculosis $327(80.1 \%)$. Likewise $359(84.4 \%)$ participants agreed that controlling of HIV/AIDS is important in controlling process of TB infection. Study participants were able to identify the common means of HIV transmission and agreed that HIV/AIDS is not a curable disease. $87.9 \%$ of participants believed that they were at risk of getting a virus in the past, while the rest did not (Table 2). However, still there is a misconception on transmission of HIV/AIDS by mosquito bite accounting for $14.8 \%$ and wearing shared clothes (7.1\%) with their friends.

3.3. Knowledge of Study Participants on Provider Initiated HIV Testing and Counseling (PITC) Services. The majority of the study participants had heard about PITC before the time of data collection and the primary health workers were cited as a source of this information accounting for $98.3 \%$ followed by friends (43.7\%) (Figure 1). 
TABLE 1: Sociodemographic characteristics of tuberculosis patients in East Wollega administrative zone at selected health centers, August $2010(N=406)$.

\begin{tabular}{|c|c|c|}
\hline Variables & Frequency & Percentage (\%) \\
\hline \multicolumn{3}{|l|}{ Residence } \\
\hline Urban & 278 & $(68.5)$ \\
\hline Rural & 128 & $(31.5)$ \\
\hline \multicolumn{3}{|l|}{ Ethnic group } \\
\hline Oromo & 391 & $(96.3)$ \\
\hline Amharic & 13 & $(3.2)$ \\
\hline Others* & 2 & $(0.5)$ \\
\hline \multicolumn{3}{|l|}{ Sex } \\
\hline Male & 234 & $(57.6)$ \\
\hline Female & 172 & $(42.4)$ \\
\hline \multicolumn{3}{|l|}{ Age } \\
\hline 19-34 & 236 & $(58.1)$ \\
\hline $35-50$ & 125 & $(30.8)$ \\
\hline$>50$ & 45 & $(11.1)$ \\
\hline \multicolumn{3}{|l|}{ Religion } \\
\hline Orthodox & 277 & $(68.2)$ \\
\hline Protestants & 120 & $(29.6)$ \\
\hline Others $^{* *}$ & 9 & $(2.2)$ \\
\hline \multicolumn{3}{|l|}{ Marital status } \\
\hline Single & 80 & $(19.7)$ \\
\hline Married & 257 & $(63.3)$ \\
\hline Divorced/widowed/separated & 69 & $(17.0)$ \\
\hline \multicolumn{3}{|l|}{ Educational status } \\
\hline Not formal education & 230 & $(56.7)$ \\
\hline Formal education & 176 & $(43.3)$ \\
\hline \multicolumn{3}{|l|}{ Occupational status } \\
\hline Government employee & 37 & $(9.1)$ \\
\hline Farmer & 130 & $(32.0)$ \\
\hline House wife & 113 & $(27.8)$ \\
\hline Others*** & 126 & $(31.0)$ \\
\hline \multicolumn{3}{|l|}{ Family size } \\
\hline$\leq 4$ & 236 & $(58.1)$ \\
\hline$>4$ & 170 & $(41.9)$ \\
\hline
\end{tabular}

3.4. HIV Testing and Acceptance of Provider Initiated HIV Testing and Counseling Services. Of all the study participants 399 (98.2\%) are initiated for HIV test by their TB treatment supervisor during TB treatment follow-up; of those, 369 (92.5\%) TB patients accepted the initiation and 353 (95.7\%) patients had undergone HIV test and received their test result. On the other hand, about 30 (7.5\%) patients did not accept the initiation because of different reasons like waiting until $\mathrm{TB}$ treatment completion, unable to cope following positive result and the remaining $7(1.8 \%)$ participants are not encouraged for PITC. 357 (87.9\%) did not know their serostatus about HIV before the study period; of those 92 (25.5\%) TB patients were willing to check their status for HIV in the past after advice was given. According to document review result the prevalence of HIV among TB patients who
TABLE 2: Knowledge related to TB/HIV/AIDS among tuberculosis patients in East Wollega administrative zone at selected health centers, August 2010, $(N=406)$.

\begin{tabular}{|c|c|c|}
\hline TB/HIV/AID related questions & Frequency & $(\%)$ \\
\hline \multicolumn{3}{|l|}{ Source of $\mathrm{TB}^{\#}$} \\
\hline From TB patients & 402 & $(99.0)$ \\
\hline Polluted air & 381 & $(93.8)$ \\
\hline Contaminated water & 81 & $(20.0)$ \\
\hline Lump of earth & 93 & $(22.9)$ \\
\hline Others* & 8 & $(1.90)$ \\
\hline \multicolumn{3}{|l|}{ Risk of acquiring $\mathrm{TB}^{\#}$} \\
\hline Poor people & 328 & $(80.8)$ \\
\hline Living with TB patient & 380 & $(93.6)$ \\
\hline Having HIV/AIDS & 306 & $(75.4)$ \\
\hline \multicolumn{3}{|l|}{$\begin{array}{l}\text { TB increased after the era of } \\
\text { HIV/AIDS }\end{array}$} \\
\hline Increase & 327 & $(88.4)$ \\
\hline No difference & 79 & $(11.6)$ \\
\hline \multicolumn{3}{|l|}{$\begin{array}{l}\text { Controlling of HIV/AIDS is important } \\
\text { in TB control }\end{array}$} \\
\hline Yes & 359 & $(88.4)$ \\
\hline No & 47 & $(88.4)$ \\
\hline \multicolumn{3}{|l|}{ Route of HIV transmission ${ }^{\#}$} \\
\hline Sexual contact & 406 & $(100)$ \\
\hline Mother to child & 402 & $(99.0)$ \\
\hline Transfusion of blood & 404 & $(99.5)$ \\
\hline $\begin{array}{l}\text { Sharing of sharp materials with } \\
\text { PLHIV }\end{array}$ & 406 & $(100)$ \\
\hline Blood contact & 402 & $(99.0)$ \\
\hline Mosquito bite & 60 & $(14.8)$ \\
\hline Share wearing clothes & 29 & $(7.40)$ \\
\hline Others $^{* *}$ & 3 & $(0.70)$ \\
\hline \multicolumn{3}{|l|}{ Methods of HIV prevention ${ }^{\#}$} \\
\hline $\begin{array}{l}\text { Limiting sex to one uninfected } \\
\text { partner }\end{array}$ & 404 & $(99.5)$ \\
\hline Abstain from sexual intercourse & 401 & $(98.8)$ \\
\hline $\begin{array}{l}\text { Use of condom during sexual } \\
\text { intercourse }\end{array}$ & 392 & $(96.6)$ \\
\hline \multicolumn{3}{|l|}{ Comprehensive knowledge of HIV } \\
\hline Knowledgeable & 338 & $(83.3)$ \\
\hline Not knowledgeable & 68 & $(16.7)$ \\
\hline \multicolumn{3}{|l|}{ Self-perceived risk of HIV infection } \\
\hline No risk & 48 & $(11.8)$ \\
\hline Risk & 358 & $(88.2)$ \\
\hline
\end{tabular}

\#Multiple responses; others ${ }^{*}$ : sexual intercourse, evil spirit; others ${ }^{* *}$ : shaking hand, sharing meal.

underwent HIV counseling and testing from October 8, 2009, to August 18, 2010, was 137 (33.7\%).

3.5. Analysis of Factors Associated with Acceptance of PITC among TB Patients. In bivariate logistic regression analysis, 
TABLE 3: Bivariate and multiple logistic regression analysis of factors associated with acceptance of PITC $(n=369)$.

\begin{tabular}{|c|c|c|c|c|c|}
\hline Variables & Accepter & Not accepter & OR $(95 \% \mathrm{CI})$ & $\operatorname{AOR}(95 \% \mathrm{CI})$ & $P$ value \\
\hline Educational status & & & & & 0.03 \\
\hline Not formal education ${ }^{\mathrm{R}}$ & 200 & 26 & 1.0 & 1.0 & \\
\hline Formal education & 169 & 4 & $5.49(1.88-16.05)$ & $3.51(1.13-10.91)$ & \\
\hline Controlling of HIV could help in control of TB & & & & & 0.04 \\
\hline $\mathrm{No}^{\mathrm{R}}$ & 36 & 10 & 1.0 & 1.0 & \\
\hline Yes & 333 & 20 & $4.63(2.01-10.64)$ & $3.89(1.54-9.83)$ & \\
\hline Comprehensive knowledge on HIV/AIDS & & & & & 0.011 \\
\hline Not knowledgeable $\mathrm{R}^{\mathrm{R}}$ & 49 & 13 & 1.0 & 1.0 & \\
\hline Knowledgeable & 320 & 17 & $4.99(2.84-10.92)$ & $3.22(1.3-7.97)$ & \\
\hline Self-perceived risk of contracting HIV & & & & & 0.028 \\
\hline No risk ${ }^{\mathrm{R}}$ & 38 & 9 & 1.0 & 1.0 & \\
\hline Risk & 331 & 21 & $3.73(1.6-8.73)$ & $2.93(1.12-7.66)$ & \\
\hline Knowledge of PITC & & & & & 0.017 \\
\hline Not knowledgeable ${ }^{\mathrm{R}}$ & 5 & 3 & 1.0 & 1.0 & \\
\hline Knowledgeable & 364 & 27 & $8.09(1.83-35.67)$ & $7.56(1.14-40.35)$ & \\
\hline
\end{tabular}

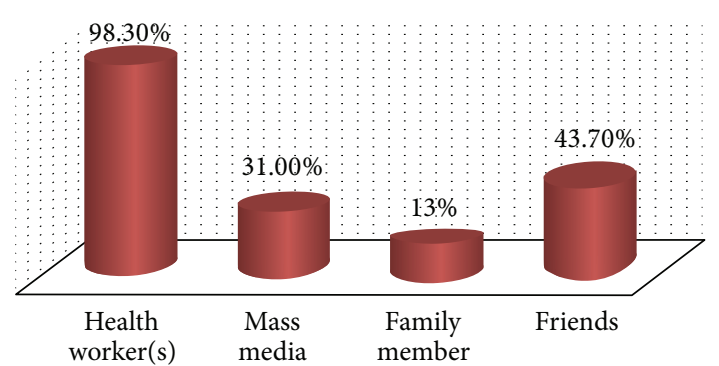

FIGURE 1: Source of information about PITC, among TB patients in East Wollega administrative zone at selected health centers, August 2010.

each explanatory variable with the outcome variable (acceptance of PITC) was assessed for its association. Variables with $P$ value $>0.3$ were not imported to multiple logistic regression for further analysis. Participants with formal education were 3.51 times more likely to accept PITC following their TB treatment supervisor initiation, as compared with who had not formal education ( $\mathrm{AOR}=3.51,95 \% \mathrm{CI}$ : $1.13-10.91$ ). Similarly, those who had comprehensive knowledge about HIV/AIDS way of transmission were 3.22 times more likely to accept PITC than who had not $(\mathrm{AOR}=3.22,95 \% \mathrm{CI}: 1.3-7.97)$. Who understand the importance of controlling HIV/AIDS helps in controlling of TB were 3.89 times more likely to accept PITC than those who do not know (AOR $=3.89,95 \%$ CI: 1.54-9.83) (Table 3).

\section{Discussion}

Most of the TB patients were initiated for HIV test by their TB treatment supervisors 399 (98.7\%) during TB treatment follow-up. Among those initiated 369 (92.5\%) accepted PITC and of those $353(95.7 \%)$ were tested and received their test result. In this study there is a high acceptance of PITC (92.5\%) among TB patients. The study results that were not comparable with the previous studies conducted in Addis Ababa (capital city of Ethiopia) and Arba Minch (south nation, nationality people) among TB patients showed $66.6 \%$ and $73 \%$, respectively $[13,14]$. This difference may relate to the recent introduction of free life-saving antiretroviral therapy at selected health centers throughout Ethiopia. Similarly, this finding was comparable with studies done in India, Kenya, Cameroon, and Congo which showed 97\%, 91.5\%, 94.5\%, and $96.25 \%$, respectively $[15,16]$. The reason for increment number of acceptances was that those patients who refuse to undergo an HIV test at the beginning of TB treatment may accept PITC initiation during TB treatment follow-up.

According to documents reviewed result, the prevalence of HIV among TB patients who undergo HIV test was $33.7 \%$. This result is comparable with studies done in North West Ethiopia and South Ethiopia [17-19]. However, this finding is not in line with the Federal HIV/AIDS prevention and a control office report in March 2010 which accounts for $20 \%$ [17]; the reason for this might be that the increment of awareness and knowledge on TB-HIV infection and PITC services during TB treatment follow-up may increase the chance of positivity.

Patients who had self-perceived risk of contracting HIV/AIDS accept PITC as compared to not self-perceived risk patients $(\mathrm{OR}=3.7395 \%, \mathrm{CI}$ : $1.6-8.73)$. This result was in agreement with a study done in Arba Minch hospital among TB patients that showed self-perceived risk patients were five times more willing to accept the initiation than nonself-perceived risk patients [20]. The possible explanation for this may be those patients who considered themselves at risk of getting HIV in the past are more interested to know their serostatus in order to benefit from the available treatment options related to HIV including provision of ART as compared to not self-perceived risk individuals (Table 3). Similarly, patients who had knowledge about provider initiated HIV testing and counseling service were more likely to 
accept PITC than unknowledgeable patients ( $\mathrm{OR}=8.09,95 \%$, CI: 1.83-35.67). This finding is in agreement with study done in North West Ethiopia [17].

Generally, in this study there is a high acceptance of PITC among TB patients following the initiation of TB treatment supervisor. This is important for the control, prevention, and treatment of TB-HIV coinfection strategies. Despite the high knowledge of TB/HIV, still there is a misconception about TB/HIV means of transmission. Therefore, information or health education about TB and HIV, particularly on way of transmission, prevention, and control, should be provided intensively by health extension workers during home-tohome visiting and TB treatment supervisors provide counseling regularly for all forms of TB patients.

\section{Conflict of Interests}

The authors declare that they have no competing interests.

\section{Authors' Contribution}

Wakjira Kebede conceived the study, undertook statistical analysis, and drafted the paper. Fikru Keno, Gutu Mamo, and Temesgen Ewunetu were involved in data collection and data entry. All authors contributed to the writing of the paper and approved the submitted version of the paper.

\section{Acknowledgments}

The authors acknowledge the Zonal Health Bureau of East Wollega for providing important information. Their acknowledgment is also for all health center staff members for their technical assistance. Finally, their thanks also go to all the study participants for their trust and cooperation.

\section{References}

[1] WHO, "Joint newsletter of the TB/HIV and MDR-TB working groups of the stop TB partnership," 2012, http://www.stoptb .org/wg/tb_hiv/.

[2] WHO, Tuberculosis Control, Epidemiology Strategy and Financing Report, World Health Organization, Geneva, Switzerland, 2009.

[3] V. M. Vashishtha, "WHO Global Tuberculosis Control Report 2009: tuberculosis elimination is a distant dream," Indian Pediatrics, vol. 46, no. 5, pp. 401-402, 2009.

[4] WHO, Networking for Policy Change, TB/HIV Participant's Guide, 2007.

[5] WHO, "Stop TB partnership," Tuberculosis facts, 2009, http:// www.who.int/tb/.

[6] Ministry of Health, Health and Health Related Indicators, Planning and Programming Department, FMOH, Addis Ababa, Ethiopia, 2004.

[7] E. L. Corbett, C. J. Watt, N. Walker et al., "The growing burden of tuberculosis: global trends and interactions with the HIV epidemic," Archives of Internal Medicine, vol. 163, no. 9, pp. 1009-1021, 2003.

[8] Federal Ministry of Health, TB/HIV Implementation Guideline, Federal Ministry of Health, Addis Ababa, Ethiopia, 2005.
[9] WHO/UNAIDS, Guidance on Provider-Initiated HIV Testing and Counseling in Health Facilities, World Health Organization, Geneva, Switzerland, 2007.

[10] Central Statistical Authority and ORC Macro, Demographic and Health Survey of Ethiopia, Addis Ababa: CSA and ORC Macro, Calverton, 2005.

[11] MOH, AIDS in Ethiopia, Ministry of Health, 5th edition, 2004.

[12] N. B. Njozing, "Assessing the accessibility of HIV care packages among tuberculosis patients in the Northwest Region, Cameroon," BMC Public Health, vol. 10, article 129, 2010.

[13] A. Van Rie, M. Sabue, N. Jarrett et al., "Counseling and testing TB patients for HIV: evaluation of three implementation models in Kinshasa, Congo," International Journal of Tuberculosis and Lung Disease, vol. 12, no. 3, pp. S73-S78, 2008.

[14] A. Aklilu, Assessment of Willingness of TB Patients Towards VCT in Selected Health Centers, North Gondar Administrative Zone, Addis Ababa, Ethiopia, 2005.

[15] H. Michael, C. Jira, K. Tushune, and B. Girma, "Health workers perspectives on incentive structure: the case of East wollega zone," Ethiopian Journal of Health Sciences, vol. 19, no. 3, 2009.

[16] B. E. Thomas, P. K. Dewan, S. Vijay et al., "Perceptions of tuberculosis patients on provider-initiated HIV testing and counseling-a study from south India," PLoS ONE, vol. 4, no. 12, article e8389, 2009.

[17] D. Jerene, A. Endale, and B. Lindtjørn, "Acceptability of HIV counselling and testing among tuberculosis patients in south Ethiopia," BMC International Health and Human Rights, vol. 7, article 4, 2007.

[18] A. Ayenew, A. Leykun, R. Colebunders, and A. Deribew, "Predictors of HIV testing among patients with tuberculosis in North West Ethiopia: a case-control study," PloS ONE, vol. 5, no. 3, article e9702, 2010.

[19] M. Melaku, "Assessment of VCT utilization and willingness to accept provider-initiated HIV counseling and testing among tuberculosis patients in Addis Ababa Ethiopia," Ethio Journal, p. $5,2007$.

[20] J. Odhiambo, W. Kizito, A. Njoroge et al., "Provider-initiated HIV testing and counselling for TB patients and suspects in Nairobi, Kenya," International Journal of Tuberculosis and Lung Disease, vol. 12, no. 3, pp. S63-S68, 2008. 


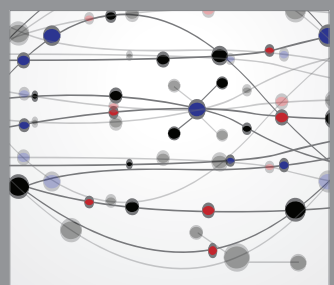

The Scientific World Journal
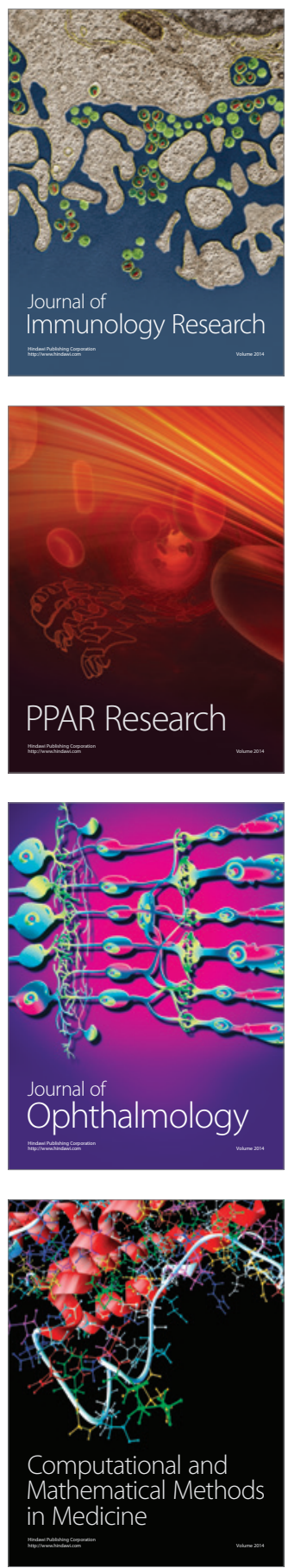

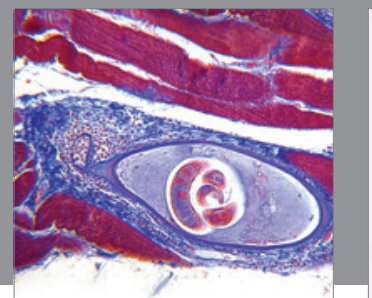

Gastroenterology

Research and Practice
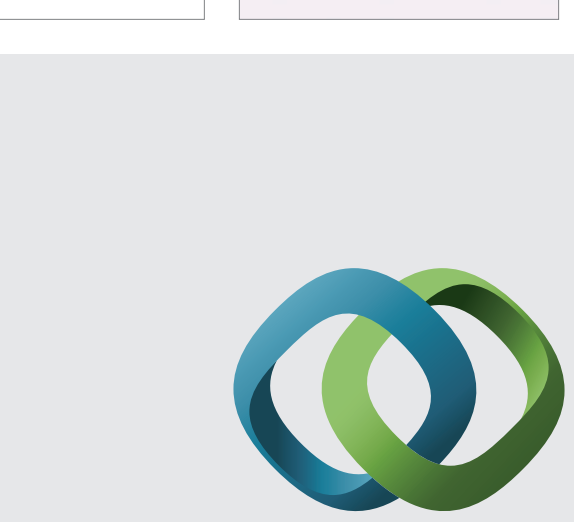

\section{Hindawi}

Submit your manuscripts at

http://www.hindawi.com
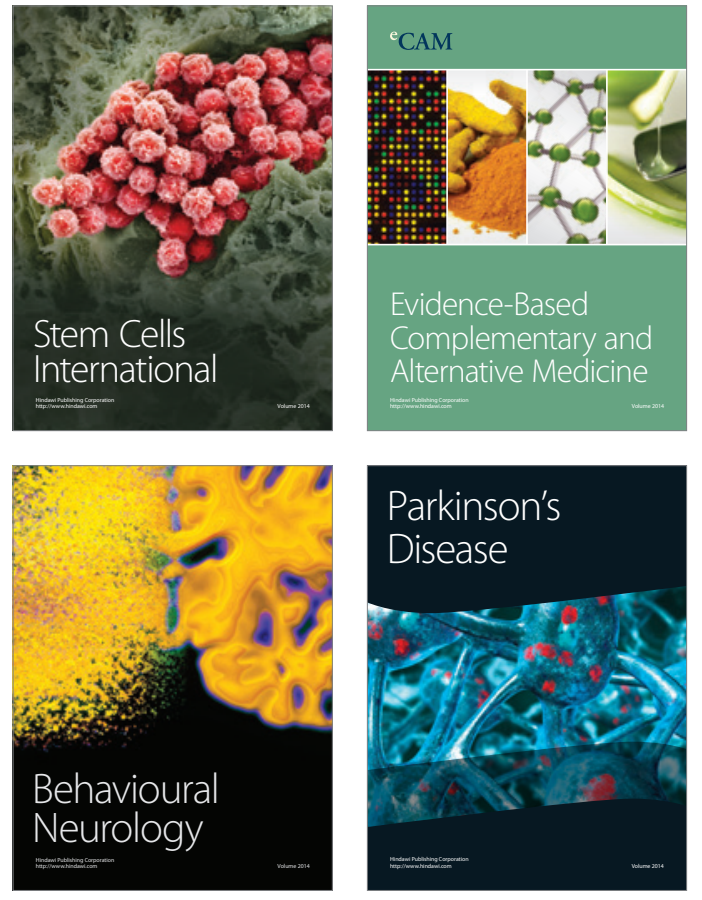
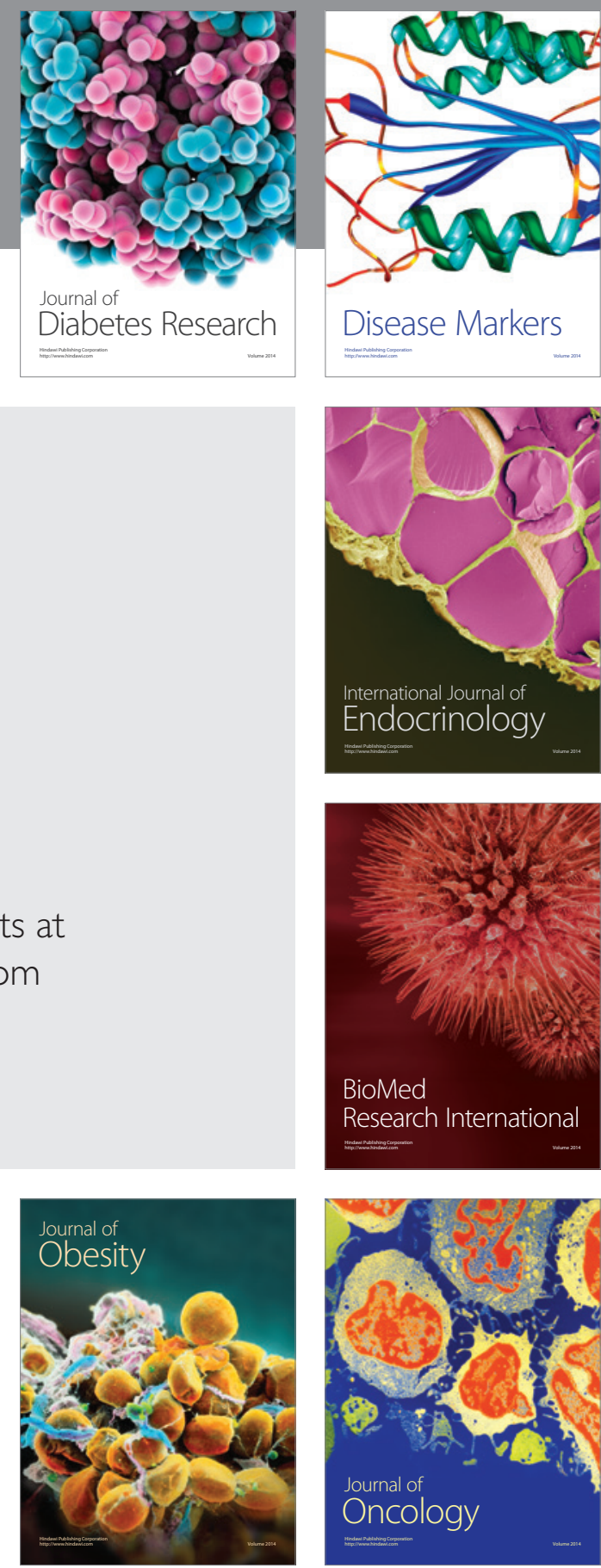

Disease Markers
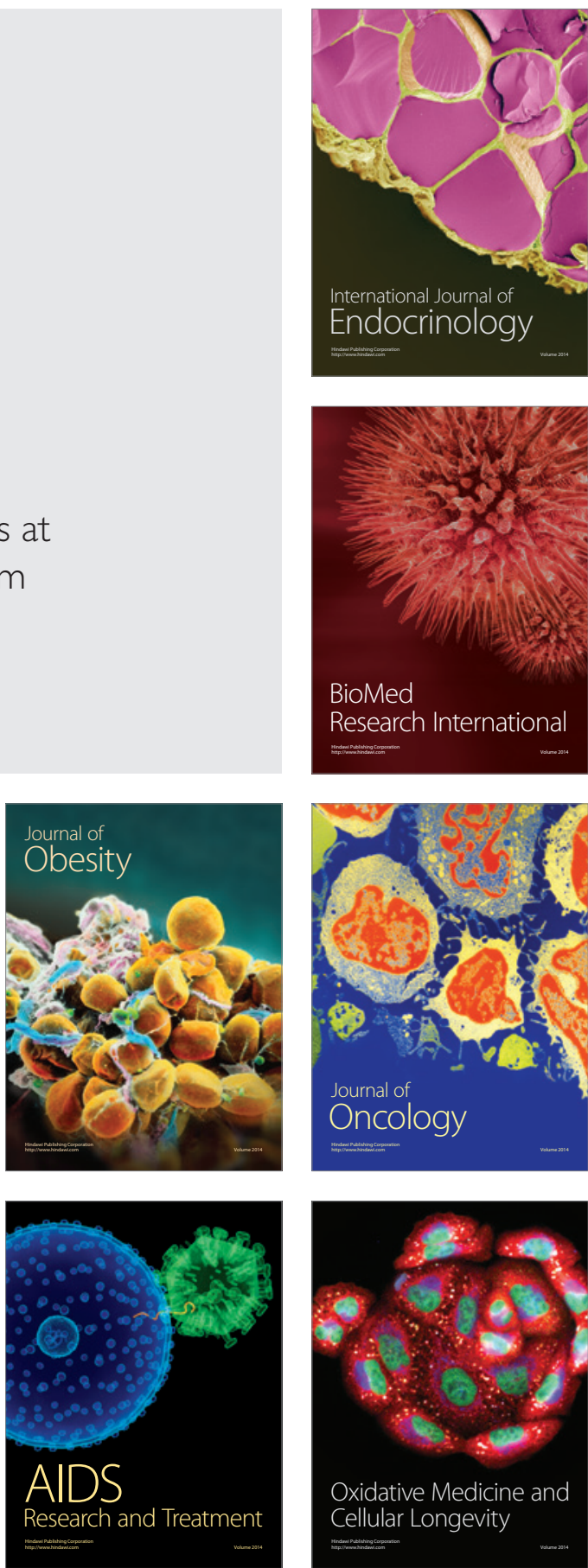